\title{
A!
}

This is an electronic reprint of the original article.

This reprint may differ from the original in pagination and typographic detail.

Zubiaga, A.; Plazaola, F.; Garcia, J.A.; Tuomisto, Filip; Munoz-San Jose, V.; Tena-Zaera, R.

\section{Positron annihilation lifetime spectroscopy of ZnO bulk samples}

Published in:

Physical Review B

DOI:

10.1103/PhysRevB.76.085202

Published: 01/08/2007

Document Version

Publisher's PDF, also known as Version of record

Please cite the original version:

Zubiaga, A., Plazaola, F., Garcia, J. A., Tuomisto, F., Munoz-San Jose, V., \& Tena-Zaera, R. (2007). Positron annihilation lifetime spectroscopy of ZnO bulk samples. Physical Review B, 76(8), 1-8. [085202].

https://doi.org/10.1103/PhysRevB.76.085202

This material is protected by copyright and other intellectual property rights, and duplication or sale of all or part of any of the repository collections is not permitted, except that material may be duplicated by you for your research use or educational purposes in electronic or print form. You must obtain permission for any other use. Electronic or print copies may not be offered, whether for sale or otherwise to anyone who is not an authorised user. 


\title{
Positron annihilation lifetime spectroscopy of $\mathrm{ZnO}$ bulk samples
}

\author{
A. Zubiaga,* F. Plazaola, and J. A. García \\ Fisika Aplikatua II Saila, Euskal Herriko Unibertsitatea, Posta Kutxatila 644, 48080 Bilbao, Spain \\ F. Tuomisto \\ Laboratory of Physics, Helsinki University of Technology, P.O. Box 1100, 02015 TKK, Espoo, Finland
}

V. Muñoz-Sanjosé and R. Tena-Zaera ${ }^{\dagger}$

Departament de Fisica Aplicada i Electromagnetisme, c/ Doctor Moliner 50, E-46100 Burjassot, Valencia, Spain

(Received 10 August 2006; revised manuscript received 20 June 2007; published 2 August 2007; corrected 6 August 2007)

\begin{abstract}
In order to gain a further insight into the knowledge of point defects of $\mathrm{ZnO}$, positron annihilation lifetime spectroscopy was performed on bulk samples annealed under different atmospheres. The samples were characterized at temperatures ranging from 10 to $500 \mathrm{~K}$. Due to difficulties in the conventional fitting of the lifetime spectra caused by the low intensity of the defect signals, we have used an alternative method as a solution to overcome these difficulties and resolve all the lifetime components present in the spectra. Two different vacancy-type defects are identified in the samples: $\mathrm{Zn}$ vacancy complexes $\left(V_{\mathrm{Zn}}-X\right)$ and vacancy clusters consisting of up to five missing $\mathrm{Zn}-\mathrm{O}$ pairs. In addition to the vacancies, we observe negative-ion-type defects, which are tentatively attributed to intrinsic defects in the $\mathrm{Zn}$ sublattice. The effect of the annealing on the observed defects is discussed. The concentrations of the $V_{\mathrm{Zn}}-X$ complexes and negative-ion-type defects are in the $0.2-2 \mathrm{ppm}$ range, while the cluster concentrations are 1-2 orders of magnitude lower.
\end{abstract}

DOI: 10.1103/PhysRevB.76.085202

PACS number(s): 61.72.Ji, 78.70.Bj, 61.82.Fk

\section{INTRODUCTION}

The fabrication of photonic devices of ultraviolet wavelength range and high frequency electronic devices requires semiconductors with a wide band gap. The physical and chemical properties of $\mathrm{ZnO}$ make it a promising candidate for optoelectronic applications in the blue-UV range. ${ }^{1} \mathrm{ZnO}$ is a semiconducting compound of the II-VI family with a direct wide band gap ( $3.37 \mathrm{eV}$ at room temperature) and it presents an exciton binding energy of $60 \mathrm{meV}^{2}$ As a consequence, the excitons are not easily thermally dissociated and the near band gap recombination is very efficient even at room temperature. In addition, the electrical and optical properties of $\mathrm{ZnO}$ are very resistant to deterioration under irradiation. ${ }^{3}$

Defects and impurities in semiconductors can trap electrons and holes. The electronic and optical properties of the devices are largely affected by the defects and their presence must be controlled. As-grown $\mathrm{ZnO}$ samples are $n$ type, but if a $p-n$ junction is to be constructed, $\mathrm{ZnO}$ bulk samples of $p$-type conductivity need to be obtained. Great effort is being put into obtaining $p$-type bulk samples. Nitrogen, ${ }^{4}$ phosphorus, ${ }^{5}$ and arsenic $^{6}$ are potential acceptor impurities, but it is difficult to obtain crystals with stable $p$ character. $^{2}$

The creation of excess donors is determined by the thermodynamics and the growth process. Many parameters, such as the partial pressure of the components or the growth temperature, control this process. In thermodynamical equilibrium, the concentration of the defects is determined by their formation energy. In subsequent thermal treatments, the autodiffusion of vacancies and interstitials can decrease their concentration. Positron annihilation lifetime spectroscopy (PALS) is a useful tool for studying open volume defects in semiconductors. ${ }^{7}$ Positrons get trapped at negative and neutral vacancy defects, and also at hydrogenic states around negatively charged defects with no open volume, such as acceptor-type impurities.
In this work, we study $\mathrm{ZnO}$ bulk crystals, grown by vapor transport (VT), annealed under different atmospheres: oxygen vapor overpressure, $\mathrm{Zn}$ vapor overpressure, and vacuum. PALS measurements have been performed from 10 to $500 \mathrm{~K}$. The origin of the observed defects and their behavior in the annealed samples are discussed.

\section{EXPERIMENT}

As said before, $\mathrm{ZnO}$ bulk samples were grown by VT under slightly Zn-rich conditions. ${ }^{8}$ The samples were annealed at $900{ }^{\circ} \mathrm{C}$ for $20 \mathrm{~h}$ in atmosphere composed of oxygen $\left(\mathrm{O}_{2}\right)$ vapor $2 \mathrm{~atm}$ overpressured, in atmosphere composed of $\mathrm{Zn}$ vapor 2 atm overpressured, and in vacuum. A couple of identical samples are necessary to measure the positron annihilation lifetime. Then, all the annealed and the as-grown samples were cut from the same ingot for a direct comparison of the differences and their characteristics.

The PALS measurements were performed with a fast-fast spectrometer in collinear geometry ${ }^{7}$ in the $10-500 \mathrm{~K}$ temperature range. The time resolution of the experimental setup was 270 ps. As positron source, we used ${ }^{22} \mathrm{NaCl}$ deposited on a thin $\mathrm{Al}$ foil, sandwiched between two sample pieces. All spectra have $(1.7-2) \times 10^{6}$ counts and the lifetime spectra were analyzed after subtracting the background and the source corrections $(215$ ps $3 \%, 400$ ps $5.1 \%, 1500$ ps $0.09 \%$ ) determined with a saturated vapor pressure (SVP)-grown reference $\mathrm{ZnO}$ sample. ${ }^{9}$ The analyses were performed with two lifetimes convoluted with the Gaussian resolution function of the experimental setup. The average lifetime $(\tau)$, which coincides with the center of mass of the spectrum, has the smallest experimental error, so the changes in the average lifetime are the best evidence of changes in the composition of the defects. It can be calculated as the sum of the indi- 
vidual lifetimes, weighted with the normalized intensity of each component: $\tau=\sum_{i} I_{i} \tau_{i}$. The increase of the average lifetime above the positron lifetime in the bulk $\left(\tau_{b}\right)$ indicates the presence of vacancy-type defects. The kinetic trapping model ${ }^{10}$ is used to fit the experimental positron lifetime data.

\section{POSITRON TRAPPING AT DEFECTS}

When a positron enters a semiconductor material, it loses its kinetic energy rapidly and reaches thermal equilibrium with the crystal lattice. It diffuses through the material until it annihilates directly from the delocalized state with an electron, or gets trapped at a defect and annihilates from a localized state. Neutral and negatively charged vacancy defects are efficient positron traps due to the missing positive ion core. Also, negatively charged defects with no open volume can trap positrons at low temperatures. The positron lifetime correlates with the open volume at the annihilation site; hence, it is longer at vacancy defects than in the free state in the lattice. In the case of neutral defects, the positron trapping is independent of the temperature, but the trapping coefficient of negatively charged defects ${ }^{10}$ varies as $T^{-1 / 2}$.

The positron trapping rate to a defect $\left(\kappa_{D}\right)$ is proportional to its concentration $\left(C_{D}\right): \kappa_{D}=\mu_{D} C_{D}$. The trapping coefficient $\left(\mu_{D}\right)$ depends on the type of the defect and the surrounding lattice. ${ }^{11}$ The trapping and annihilation processes are described by a set of kinetic equations. They relate the rate of change of density of positrons in a determined state to the trapping, detrapping, and annihilation rates at all the states of the positron. ${ }^{10}$

In addition to vacancy defects, positrons can also get trapped at hydrogenlike Rydberg states surrounding negative centers with no open volume (called ionic traps from this point forward), where their binding energy is small (typically $E_{b} \leqslant 0.1 \mathrm{eV}$ ). Positrons are not well localized at the ionic traps and their wave functions extend to the bulk of the material; hence, the annihilation characteristics are similar to the delocalized state in the lattice and they get detrapped below room temperature. ${ }^{12}$ The detrapping process from the ionic traps is thermally activated and the activation energy is equal to the binding energy $E_{b} .{ }^{13}$ The escape rate $\left(\delta_{i o n}\right)$ can be written as

$$
\delta_{\text {ion }}=\kappa_{\text {ion }} \frac{\left(2 \pi m^{*} k_{B} T / h^{2}\right)}{c_{\text {ion }}} 3 / 2 e^{\left(-E_{b} / k_{B} T\right)},
$$

where $m^{*} \simeq m_{0}$ is the positron effective mass, $k_{B}$ the Boltzmann constant, $c_{i o n}$ the ion concentration, and $h$ the Planck constant. In principle, positrons can also escape from the Rydberg states around negatively charged vacancies, but we assume that the transition from the Rydberg state to the ground state in the vacancy is fast enough so that this effect can be neglected. ${ }^{10}$ An effective trapping rate of the ionic traps can thus be defined as

$$
\kappa_{i o n}^{e f f}=\frac{\kappa_{\text {ion }}}{1+\delta_{\text {ion }} / \lambda_{\text {ion }}},
$$

where $\lambda_{\text {ion }}=\lambda_{b}=\tau_{b}^{-1}$ is the annihilation rate of positrons trapped at the Rydberg state, which coincides with the anni- hilation rate from the delocalized state in the lattice.

We will show in this work that the analyzed $\mathrm{ZnO}$ samples contain two different types of vacancy defects (Zn vacancy complex defects, $V_{\mathrm{Zn}^{-}} X$, and vacancy clusters) and ionic traps. In this case, the average lifetime is related to the positron trapping rates and annihilation lifetimes in the four different states as follows:

$$
\tau=\tau_{b}+\frac{\kappa_{v}\left(\tau_{v}-\tau_{b}\right)+\kappa_{c l}\left(\tau_{c l}-\tau_{b}\right)}{\frac{1}{\tau_{b}}+\kappa_{i o n}^{e f f}+\kappa_{v}+\kappa_{c l}} .
$$

At temperatures high enough, positrons escape efficiently from the ionic traps and the experimental values can be analyzed with the assumption that only vacancy-type defects contribute to the data. In addition, if only one vacancy type contributes to the annihilation spectra, the annihilation parameters can be estimated using the equations of the onevacancy-type defect trapping model:

$$
\begin{gathered}
\kappa_{v}=\frac{1}{\tau_{b}} \frac{\tau-\tau_{b}}{\tau_{v}-\tau}, \\
\tau_{1}^{*}=\frac{\tau_{b}}{1+\tau_{b} \kappa_{v}},
\end{gathered}
$$

where $\tau_{v}=\tau_{2}$, the longer lifetime in the decomposition of the lifetime spectra.

The trapping rate at ionic traps $\left(\kappa_{\text {ion }}\right)$ can be calculated at low temperatures, where the escape rate is small enough to be neglected, using the following relation:

$$
\kappa_{\text {ion }} \simeq \kappa_{\text {ion }}^{e f f}=\frac{\kappa_{v}\left(\tau_{v}-\tau\right)+\kappa_{c l}\left(\tau_{c l}-\tau\right)+\lambda_{b}\left(\tau_{b}-\tau\right)}{\tau-\tau_{i o n}} .
$$

Due to finite time resolution, annihilations at source material, and background noise, usually only one or two lifetime components are resolved in the experimental spectrum. The longest lifetime of the decomposition corresponds to the lifetime of positrons trapped at vacancy-type defects. If the lifetime difference of two annihilation states is not large enough $(\Delta \tau \sim 20 \%-30 \%)$, they will appear mixed in a single lifetime component in the fitting. The shortest lifetime is a superposition of the positron lifetime in the delocalized state, that at the ionic traps, and that at the vacancy-type defects.

\section{RESULTS}

Figure 1 shows the average lifetime measured at temperatures ranging from 10 to $500 \mathrm{~K}$. A SVP-grown reference sample has also been measured at $300 \mathrm{~K}$. The vacancy concentration in this sample is very low, and at room temperature only annihilations in the bulk are observed, giving an average positron lifetime of $171 \mathrm{ps}$, in good agreement with the lifetime measured in this sample at room temperature in Ref. 9. The average positron lifetime in all the VT-grown samples is above the bulk lifetime. This is a clear indication that positrons are trapped at vacancy defects. The average positron lifetime in the $\mathrm{Zn}$ annealed sample is 5-6 ps lower 


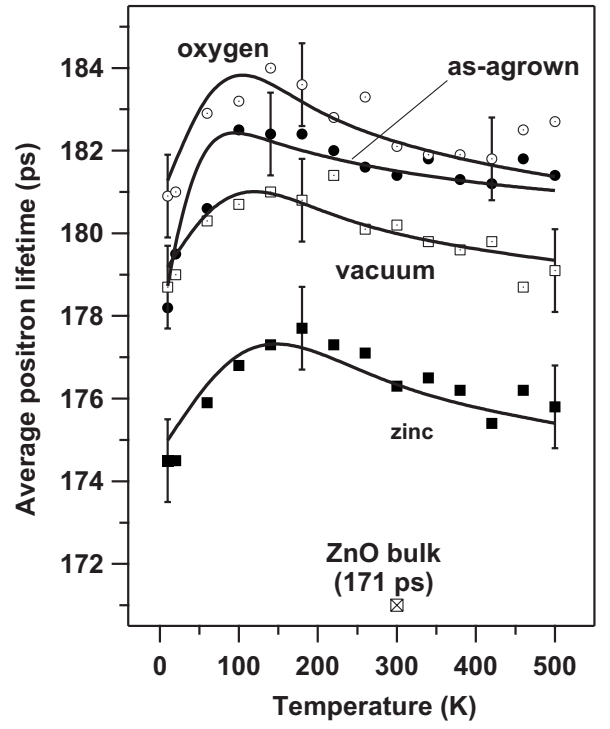

FIG. 1. The average positron lifetime in the $\mathrm{ZnO}$ samples annealed under different conditions, measured in the temperature range from 10 to $500 \mathrm{~K}$. Also, the bulk lifetime measured in the SVP-grown reference sample is shown. The solid lines show the average positron lifetime fitted with the kinetic trapping model.

than that in the as-grown sample in the whole measurement temperature range, and the $\mathrm{Zn}$ annealed sample has the lowest lifetime values among the measured samples. The average lifetime in the oxygen annealed sample is 3 ps higher than that in the as-grown sample at $10 \mathrm{~K}$, but it is only $1 \mathrm{ps}$ higher at $500 \mathrm{~K}$. On the other hand, the average lifetime in the vacuum annealed sample is similar to that measured in the as-grown sample at $10 \mathrm{~K}$ and 2 ps lower at $500 \mathrm{~K}$.

The decrease of the average positron lifetime with increasing temperature (Fig. 1) above $200 \mathrm{~K}$ is an indication of the vacancy defects being in the negative charge state. This behavior is due to the $T^{-1 / 2}$ dependence of the positron trapping coefficient to negative centers. Below $200 \mathrm{~K}$, the average positron lifetime decreases strongly with decreasing temperature. This is explained by the enhancement of positron trapping at negative-ion-type defects (with no open volume), from which the positron escapes at higher temperatures.

Figure 2 shows the results obtained by fitting the spectra with two lifetimes as free parameters. If we focus on temperatures above $200 \mathrm{~K}$, we can observe that the values of the higher lifetime component $\left(\tau_{2}\right)$ and its intensity $\left(I_{2}\right)$ in all the measured samples are almost similar within the experimental error. Also, the lower lifetime components $\left(\tau_{1}\right)$ in the asgrown, the oxygen annealed, and the vacuum annealed samples have similar values. However, $\tau_{1}$ in the sample annealed under $\mathrm{Zn}$ atmosphere is shorter over the whole temperature range. The differences in the lifetime components between the samples at temperatures below $200 \mathrm{~K}$ are larger.

The value of $\tau_{2}$ is clearly larger than the values reported for the $\mathrm{Zn}$ vacancy. It ranges between 300 and $350 \mathrm{ps}$ for temperatures higher than $200 \mathrm{~K}$ and $\tau_{2} / \tau_{b}$ is around 1.7-2.0. This value is higher than the typical value for monovacancies in semiconductors. ${ }^{10,14}$ Hence, defects with larger open volume, i.e., vacancy clusters, contribute to $\tau_{2}$.

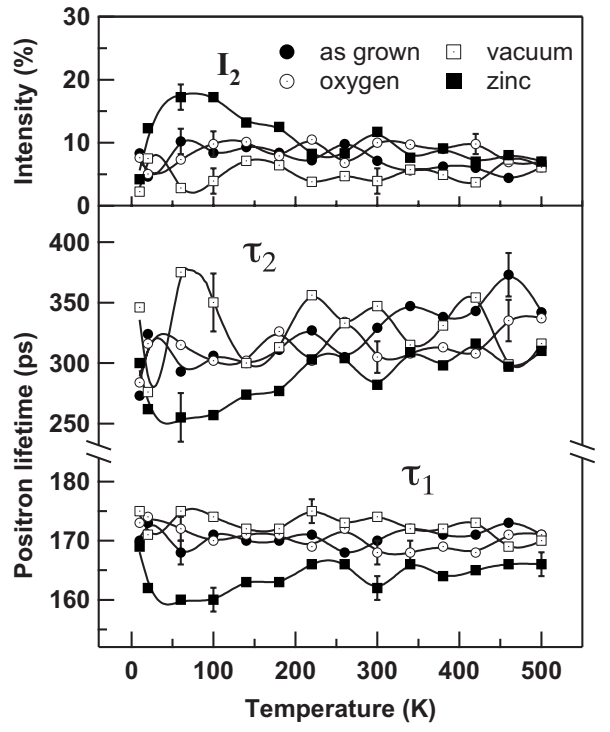

FIG. 2. Decomposition of the lifetime of all measured samples in the full temperature range.

\section{DISCUSSION}

\section{A. Positron lifetime in the $\mathrm{ZnO}$ lattice}

Results of positron measurements in bulk $\mathrm{ZnO}$ crystals grown by EaglePicher (EP), the material used as reference in this work, have been reported earlier by three different groups. ${ }^{9}, 15,16$ The conclusions in these works coincide: the vacancy defect concentration in this material is below the detection limit of positron annihilation spectroscopy and only one lifetime component can be fitted to the spectrum at room temperature, thus giving directly the bulk lifetime. Hence, the EP ZnO samples are ideal for use as a reference. The bulk lifetime values reported by Brunner et al. ${ }^{15}$ and Uedono et al. ${ }^{16}$ (161 and $158 \mathrm{ps,} \mathrm{respectively)} \mathrm{are} \mathrm{slightly}$ lower than that reported by Tuomisto et al. ${ }^{9}$ (171 ps). However, slight differences in the positron lifetime spectrometers, such as scintillators, geometry, and electronic settings, influence the absolute values of the positron lifetimes. In addition, the source corrections used are reported only in Refs. 9 and 17, which makes the direct comparison of the absolute values even less meaningful. Very recently, ${ }^{18}$ a bulk lifetime as low as 151 ps was also reported (in melt-grown material) from a lifetime spectrum with $1 \times 10^{6}$ counts measured at room temperature in a setup with a resolution of $250 \mathrm{ps}$, but this value was estimated from a lifetime spectrum dominated by a vacancy-related lifetime of 257 ps (intensity of 60\%), and as such it is difficult to compare in a straightforward way with directly measured values. The source corrections used were not given in this report either.

The above discussion demonstrates the importance of having high-quality (low vacancy defect concentration) material, such as the EP bulk crystal, as a reference sample for positron measurements. In addition to as-grown samples, electron-irradiated $\mathrm{ZnO}$ has also been studied by Brunner et $a l .{ }^{15}$ They report a Zn-vacancy-related lifetime of $210 \pm 10 \mathrm{ps}$, somewhat lower than the value obtained by Tuomisto et al. ${ }^{9}(230 \pm 10 \mathrm{ps})$. The important value in the 
identification of different vacancy defects is the ratio $\tau_{D} / \tau_{B}$ of the defect-related and bulk lifetimes. As the bulk lifetime measured with the setup of Brunner et al. ${ }^{15}$ is lower, the agreement between the $\mathrm{Zn}$ vacancy values obtained by the two groups is very good (the ratios are $\tau_{D} / \tau_{B}=1.30 \pm 0.05$ and $\tau_{D} / \tau_{B}=1.35 \pm 0.05$, respectively). In addition to the irradiated $\mathrm{ZnO}$, a value of $230 \pm 10$ ps has been obtained for $\mathrm{Zn}$ vacancies in as-grown material as well, ${ }^{19}$ measured with the same setup as in this work.

As the EP $\mathrm{ZnO}$ reference sample, on which all previous works by different groups agree to have a vacancy defect concentration below the PAS detection limit, gives in the setup used in the present measurements a single lifetime of $171 \mathrm{ps}$ at room temperature, this lifetime is used as the bulk lifetime in $\mathrm{ZnO}$ in this work. Consequently, $230 \mathrm{ps}$ is used as the $\mathrm{Zn}$ vacancy specific lifetime. Finally, it is worth noticing that in order to test the quality of the measured lifetimes, defect-free $\mathrm{Si}$ and GaAs standard references were measured with the same setup to give bulk lifetimes of $220 \pm 1$ and $231 \pm 1 \mathrm{ps}$, respectively. These values are in excellent agreement with the values widely reported in the literature: $\tau_{B}$ $=219-222$ ps for bulk Si (see, e.g., Ref. 20 and references therein) and $\tau_{B}=228-232 \mathrm{ps}$ for bulk GaAs (see, e.g., Ref. 21 and references therein).

\section{B. Fitting procedure}

Fitting the spectra using two lifetimes as free parameters shows that $\tau_{1}$ in the $\mathrm{Zn}$ annealed sample is systematically lower than in the other samples (Fig. 2). This feature correlates with the lower value of the average lifetime in this sample. The other parameters $\left(\tau_{2}\right.$ and $\left.I_{2}\right)$ do not show any clear difference between the $\mathrm{Zn}$ vapor annealed sample and the other samples. Moreover, $\tau_{1}$ is a statistically strong parameter due to the large value of $I_{1}(\sim 90 \%)$ in all the measured samples and temperatures.

We tested the one-vacancy trapping model by comparing the fitted $\tau_{1}$ of all the samples in the decomposition analysis of the spectra with two lifetimes as free parameters, with $\tau_{1}^{*}$ obtained from the model. Here, we used the fitted $\tau_{2}$ as the vacancy lifetime at each temperature. The agreement between the fitted $\tau_{1}$ and $\tau_{1}^{*}$ obtained from the one-vacancy model is good only in the Zn vapor annealed sample (Fig. 3). In the other samples, $\tau_{1}^{*}$ is systematically below the fitted value. This result can be well understood if the $\mathrm{Zn}$ annealed sample contains only one type of vacancy defects and the other samples contain at least two different types of vacancyrelated defects. It must be noted that $\tau_{1}$ is below $\tau_{b}$ in the $\mathrm{Zn}$ annealed sample, as expected when only one kind of vacancy is present. The same conclusion is reached by fixing the higher lifetime component to $340 \mathrm{ps}$, the average value of the fitted $\tau_{2}$.

Fitting the experimental spectra with three lifetime components (either as free or fixed) does not work due to the low intensity (total about 10\%) of the longer components. The main problems in the analysis are the rather low intensity of the higher lifetime component and the fact that different vacancy-type defects are present in the material with lifetimes in the range $170-350 \mathrm{ps}$. Hence, focusing on the ef-

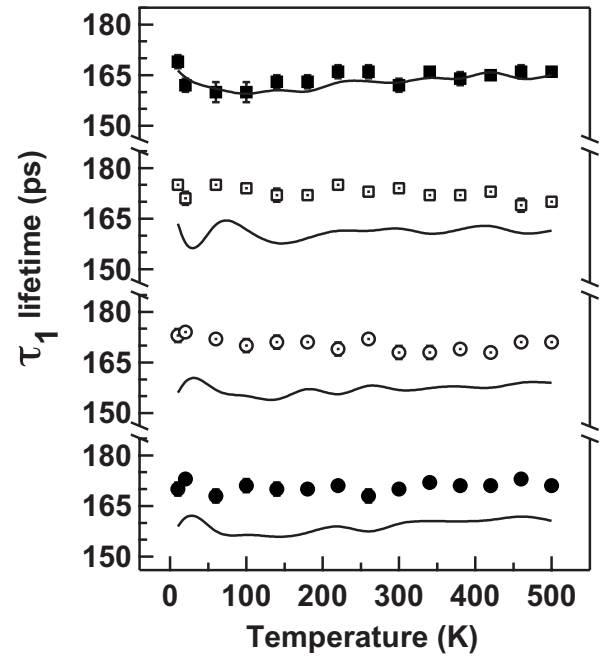

FIG. 3. The lower lifetime component $\tau_{1}$ of the figure obtained from a fit with two lifetime components as free parameters (markers) and the estimated value from the one-vacancy-type defect model $\tau_{1}^{*}$ (solid lines), using Eq. (5).

fects in $\tau_{1}$ (with intensity $\sim 90 \%$ ) is reasonable.

The above analysis indicates that three different lifetimes contribute to the annihilation spectra: the bulk lifetime of $\tau_{b} \simeq 170 \mathrm{ps}$, the lifetime specific to vacancy clusters of $\geqslant 300 \mathrm{ps}$, and a third lifetime somewhere between these two. This component is mixed to both fitted lifetimes when two lifetime components are used as free parameters.

In recent studies on bulk $\mathrm{ZnO}$ crystals, negatively charged $\mathrm{Zn}$ vacancies ${ }^{9}$ and $\mathrm{Zn}$-vacancy-related complexes in the neutral charge state ${ }^{19}$ have been observed and their lifetime was 230 ps. The improvement of the results using the onevacancy model for the fitting of the lifetime corresponding to the $\mathrm{Zn}$ vapor annealed samples suggests that the presence of $\mathrm{Zn}$ vacancies or related complexes with a lifetime of $230 \mathrm{ps}$ can be responsible for the problems in fitting the experimental lifetime with two free parameters.

Having in mind the similarities between the growth processes in this work and in Ref. 19, we can assume that $\mathrm{Zn}$ vacancies or related complexes in a neutral charge state can be present in our samples as well. Taking into account that theoretical calculations predict lower formation energy for $\mathrm{Zn}$ vacancies double negative charged than for neutral ones, ${ }^{22}$ the neutral state charge felt by positrons points toward the presence of $V_{\mathrm{Zn}}-X$ neutral complexes. Thus, $\mathrm{Zn}$ vacancies could be complexed with donor-type defects $(X)$ with no open volume or oxygen vacancies with low open volume.

As said before, the fitting with three components is not possible due to the low intensity of the higher components. Hence, assuming their neutrality, we proceed by subtracting a constant contribution as a function of temperature of the $\mathrm{Zn}$ vacancies or related complexes (lifetime $\tau_{v}=230 \mathrm{ps}$ ) from the spectra before fitting two lifetime components. Then, we optimize the subtracted intensity by comparing the fitted $\tau_{1}$ with $\tau_{1}^{*}$, which should coincide when only one-vacancy defect contributes to the spectrum. The intensity of the subtracted component is proportional to the fraction of positrons $\left(\eta_{v}\right)$ trapped at the defect responsible for this component: 


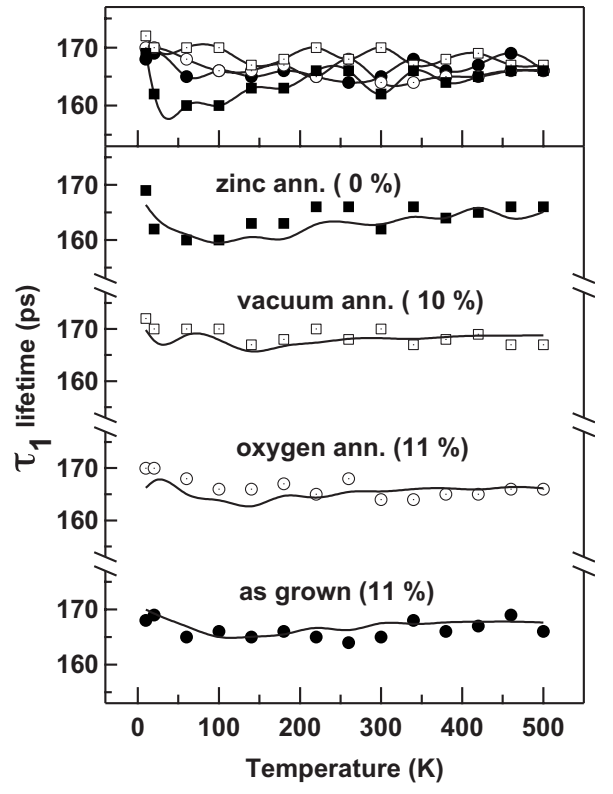

FIG. 4. A constant fraction of the $230 \mathrm{ps}$ component of the spectra has been subtracted from the spectra before obtaining lifetime components. Fit of $\tau_{1}$ using the one-vacancy-type defect model is shown. Value between parentheses is the subtracted fraction yielding the best result. In the upper graph, overlap of $\tau_{1}$ of all samples can be observed, specially at temperatures above $200 \mathrm{~K}$.

$$
I_{v}=\frac{\eta_{v}}{\tau_{v}} e^{\left(-t / \tau_{v}\right)} .
$$

The obtained best values of $\tau_{1}$ are shown in Fig. 4 in the full range of temperatures. The subtracted fraction of $230 \mathrm{ps}$ lifetime is also shown. The analysis can only be done at temperatures above $200 \mathrm{~K}$, where the effects of the ionic traps can be neglected. In the $\mathrm{Zn}$ vapor annealed sample, the best fit is obtained if the 230 ps component is not subtracted at all. If the subtracted intensity is varied around the optimal value, the comparison to the one-vacancy trapping model is weakened. This analysis supports the existence of two types of vacancy defects in the samples.

Hence, we conclude that the lifetime spectra have contributions from two types of vacancy defects at high temperatures: one of around 230 ps, associated with $\mathrm{Zn}$ vacancies or related complexes, that cannot be resolved in ordinary decompositions, and another of around 300-350 ps, associated with vacancy clusters. The $\mathrm{Zn}$ vapor annealed sample at temperatures above $200 \mathrm{~K}$ has zero intensity of the $230 \mathrm{ps}$ lifetime and positrons are trapped at one type of vacancy defects in this sample. The other samples are optimally fitted when the subtracted fractions are 9\% (vacuum annealed sample) and 10\% (as-grown and oxygen annealed samples). The procedure to arrive at the above results is as follows.

(1) The spectra are fitted using two lifetimes as free parameters, obtaining results with lower statistical noise than with three lifetimes, and a complete separation of the vacancy-type defect contributions has been obtained.

(2) The analysis is tested using the one defect trapping model.

(3) The criterion $\tau_{1}=\tau_{1}^{*}$ for the subtraction of the right intensity value is used.
TABLE I. The first column shows the trapping fractions of the 230 ps lifetime defect subtracted during the fitting of the spectra. The subsequent columns show the trapping fractions, trapping rates at $300 \mathrm{~K}$, and concentrations of $\mathrm{Zn}$ vacancies in the samples obtained by fitting the average lifetime. The concentration of the $\mathrm{Zn}$ vacancies in the $\mathrm{Zn}$ annealed sample is below the detection limit.

\begin{tabular}{lcccc}
\hline \hline & $\begin{array}{c}\text { Subtracted } \\
(\%)\end{array}$ & $\begin{array}{c}\text { Fitted } \\
(\%)\end{array}$ & $\begin{array}{c}\kappa_{v}^{0} \\
\left(\mathrm{~ns}^{-1}\right)\end{array}$ & $\begin{array}{c}C_{v} \\
(\mathrm{ppm})\end{array}$ \\
\hline As grown & 11 & 14 & $0.9(1)$ & 0.3 \\
Oxygen & 11 & 12 & $0.8(1)$ & 0.3 \\
Vacuum & 10 & 9 & $0.6(1)$ & 0.2 \\
Zn & & & & $\leqslant 0.03$ \\
\hline \hline
\end{tabular}

While making this analysis, only the $200-500 \mathrm{~K}$ temperature range has been considered, because at low temperatures positrons also annihilate at ionic traps. The presence of only one type of defect in the spectra besides the 230 ps component is necessary to apply this analysis. The subtraction of a constant intensity independent of temperature gives a very good agreement with the one-vacancy model, suggesting that the $\mathrm{Zn}$ vacancies belong to $V_{\mathrm{Zn}}-X$ neutral complexes in VT-grown $\mathrm{ZnO}$.

\section{Concentrations of the defects}

The above discussion shows that above $200 \mathrm{~K}$, positrons annihilate mainly at two positron traps: the $\mathrm{Zn}$ vacancy or related complexes $\left(\tau_{V}=230 \mathrm{ps}\right)$ and a vacancy cluster with a longer lifetime. The lifetime of positrons trapped at vacancy clusters can be estimated as an average of the values of $\tau_{2}$ obtained after the subtraction of the 230 ps component, $\tau_{c l}$ $=\tau_{2}=340 \pm 40 \mathrm{ps}$. In this temperature range, the effects of ionic traps and the variations due to the possible temperature dependence of the trapping rates are very small.

Further support of the fact that the $\mathrm{Zn}$ vacancies belong to $V_{\mathrm{Zn}}-X$ neutral complexes is the temperature variation of the average positron lifetime above $200 \mathrm{~K}$ in the $\mathrm{Zn}$ vapor annealed sample, where no $\mathrm{Zn}$ vacancies are present. Hence, the vacancy clusters are in the negative charge state. As the fitting of three lifetimes to the spectra is uncertain, we fit the average lifetime vs temperature data in the way described in the following [using Eq. (3)].

First, the high temperature range $(200-500 \mathrm{~K})$ is fitted considering two vacancy lifetimes: one of 230 ps and another of $340 \mathrm{ps}$, the effect of ionic traps being negligible at these temperatures. Next, the entire temperature range will be fitted using the trapping rates at vacancies obtained in the first step as fixed parameters to obtain the trapping rate, the concentration, and the binding energy of the ionic traps.

The values obtained after fitting the high temperature range are summarized in Tables I and II. $V_{\mathrm{Zn}}-X$ neutral complexes were fitted with a constant trapping rate $\left(\kappa_{v}=\kappa_{v}^{0}\right)$ and the vacancy clusters with $\kappa_{c l}=\kappa_{c l}^{0}(T / 300 \mathrm{~K})^{-0.5}$, as corresponds to negatively charged defects. The concentrations of $V_{\mathrm{Zn}}-X$-complex-type defects were calculated using the typical trapping coefficient for neutral vacancies in 
TABLE II. Trapping rates at $300 \mathrm{~K}$ and the concentrations of vacancy clusters obtained by fitting $\tau$ in Eq. (3), with trapping rates as fitting parameters.

\begin{tabular}{lcc}
\hline \hline & $\begin{array}{c}\kappa_{c l}^{0} \\
\left(\mathrm{~ns}^{-1}\right)\end{array}$ & $\begin{array}{c}C_{c l} \\
(\mathrm{ppm})\end{array}$ \\
\hline As grown & $0.10(2)$ & $0.010(2)$ \\
Oxygen & $0.18(3)$ & $0.018(3)$ \\
Vacuum & $0.14(4)$ & $0.014(4)$ \\
Zn & $0.17(2)$ & $0.017(2)$ \\
\hline \hline
\end{tabular}

semiconductors, ${ }^{7} \mu_{v}^{0}=2 \times 10^{15} \mathrm{~s}^{-1}$. The trapping coefficient of vacancy clusters can be estimated ${ }^{23}$ as $\mu_{c l}^{0}=N \mu_{v}^{0}$, where $N \simeq 5$ is the number of $\mathrm{Zn}-\mathrm{O}$ pairs in the cluster, giving $\mu_{c l}^{0}$ $=1 \times 10^{16} \mathrm{~s}^{-1}$.

The concentration of $V_{\mathrm{Zn}^{-}} X$ neutral complexes in $\mathrm{Zn}$ annealed sample is below the detection limit of about $0.03 \mathrm{ppm}$. In the oxygen and vacuum annealed samples, the $V_{\mathrm{Zn}}-X$ complexes are practically unaffected by the annealings, in good agreement with recent work on chemical vapor transport (CVT) $\mathrm{ZnO}$ oxygen annealed samples. ${ }^{19}$ In contrast, the concentration of vacancy clusters is higher in all the annealed samples than in the as-grown sample. It is worth noticing that the trapping coefficient at $300 \mathrm{~K}$ is higher for vacancy clusters than for $V_{\mathrm{Zn}}-X$ complexes. In addition, the clusters are in the negative charge state; as a consequence, the detection limit is lower than that of the $V_{\mathrm{Zn}}-X$ complexes, about $0.002 \mathrm{ppm}$.

Assuming that the vacancy clusters are negatively charged and the $V_{\mathrm{Zn}}-X$ complexes are neutral yields the best fit of the average lifetime in the $200-500 \mathrm{~K}$ temperature range. In addition, we want to point out that the values of the trapping fractions of the $230 \mathrm{ps} \mathrm{Zn}$ vacancy component obtained by subtracting the component when fitting the lifetime spectra are consistent with the results obtained by fitting the average lifetime vs temperature data. In addition, the low concentration (below detection limit) of $V_{\mathrm{Zn}}-X$ complexes in the $\mathrm{Zn}$ vapor annealed sample is observed with both fitting methods.

The average positron lifetime in all the samples decreases with decreasing temperature below $200 \mathrm{~K}$. This indicates that positrons are efficiently trapped at ionic traps at low temperatures. The estimated values of $\kappa_{i o n}[\mathrm{Eq}$. (6)] are shown in Fig. 5 for temperatures lower than $200 \mathrm{~K}$. The trapping rates follow a power law behavior at temperatures below $60-70 \mathrm{~K}$. At higher temperatures, they decrease rapidly because the detrapping from the defects becomes important. It is remarkable that a similar temperature dependence is obtained in all samples, as it would be the case if the same type of defects was present. The power law coefficient takes values between -0.7 and -0.8 (see Table III), in rather good agreement with both the theoretical value of -0.5 (Ref. 13) and experimental values ranging from -0.3 to -0.7 obtained in $\mathrm{GaN}$ and $\mathrm{ZnO} .{ }^{24}$

The trapping rates and concentrations of the ionic traps are presented in Table III. It is clearly seen that in all the samples, the majority of positrons annihilate as trapped at ionic traps at low temperatures: for example, in the as-grown sample $\kappa_{v}=0.9(1) \mathrm{ns}^{-1}, \kappa_{c l}=0.6(1) \mathrm{ns}^{-1}$, and $\kappa_{\text {ion }}$

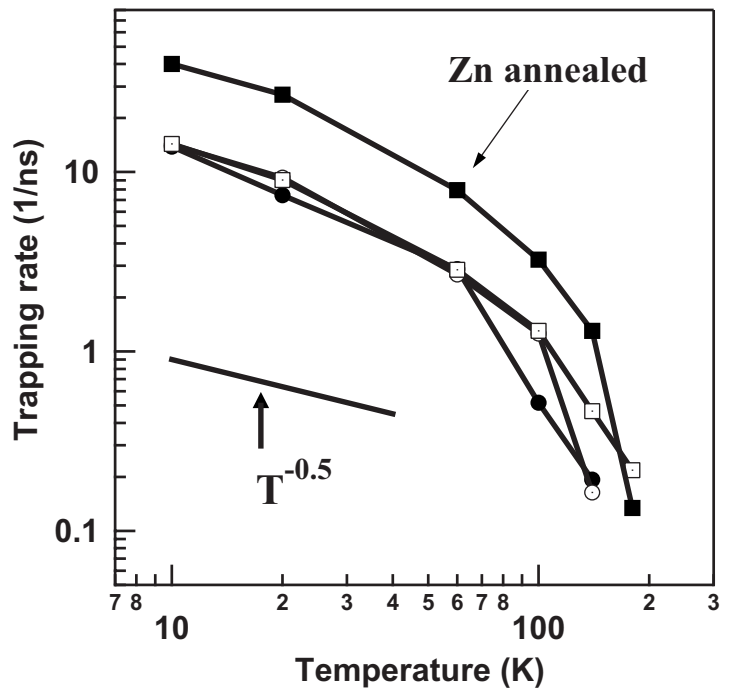

FIG. 5. Logarithmic representation of the estimated $\kappa_{\text {ion }}$ for temperatures below $200 \mathrm{~K}$. The power law behavior is followed up to $60-70 \mathrm{~K}$, where detrapping is negligible. The line representing the theoretical $T^{-0.5}$ power law behavior of negatively charged defects is also shown.

$=14(4) \mathrm{ns}^{-1}$. The values of $\kappa_{\text {ion }}$ at $10 \mathrm{~K}$ (see Table III) are used in the fit of the annihilation parameters of the ionic traps. The trapping rates of the vacancy-type defects are also introduced as fixed parameters. The average lifetime has been fitted in the entire temperature range using Eq. (3). The fitted parameters are the binding energy of positrons at ionic traps $\left(E_{b}\right)$ and the concentration of ions $\left(C_{i o n}\right)$. The fitted curves are shown in Fig. 1 and the results are presented in Table III. The binding energy at the traps is $30 \pm 10 \mathrm{meV}$, a reasonable value if we consider that nearly all positrons are detrapped at $200 \mathrm{~K}$.

It is worth noticing that the concentration of ionic traps is the highest in the $\mathrm{Zn}$ annealed sample (2 ppm obtained from the fit). The concentrations of ionic traps in the other samples are lower and similar $(0.4-0.7 \mathrm{ppm})$. The trapping coefficients of the ionic traps $\left(\mu_{i o n}=\kappa_{\text {ion }} / C_{\text {ion }}\right)$ get values in the range of $(2-3.5) \times 10^{16} \mathrm{~s}^{-1}$ at $10 \mathrm{~K}$. These values are of the same order of magnitude as the trapping coefficients obtained in GaN. ${ }^{14}$

TABLE III. The estimates for the binding energy of positrons to the ionic trap $\left(E_{b}\right)$, the power law coefficients of the trapping rate ( $n$ in $\left.T^{-n}\right)$, and the concentrations of the ionic traps $\left(C_{i o n}\right)$ obtained by fitting the average lifetime. The trapping rate to the ionic traps $\left(\kappa_{i o n}\right)$ and the estimated trapping coefficients at $10 \mathrm{~K}\left(\mu_{\text {ion }}\right)$ are also shown.

\begin{tabular}{lccccc}
\hline \hline & $\begin{array}{c}E_{b} \\
(\mathrm{meV})\end{array}$ & $n$ & $\begin{array}{c}C_{\text {ion }} \\
(\mathrm{ppm})\end{array}$ & $\begin{array}{c}\kappa_{\text {ion }} \\
\left(\mathrm{ns}^{-1}\right)\end{array}$ & $\begin{array}{c}\mu_{\text {ion }} \\
\left(\times 10^{16} \mathrm{~s}^{-1}\right)\end{array}$ \\
\hline As grown & $30(10)$ & $-0.8(1)$ & $0.4(2)$ & $14(4)$ & 3.5 \\
Oxygen & $30(10)$ & $-0.7(1)$ & $0.7(3)$ & $14(4)$ & 2.0 \\
Vacuum & $30(10)$ & $-0.8(1)$ & $0.6(3)$ & $14(5)$ & 2.3 \\
Zn & $30(10)$ & $-0.7(1)$ & $2.0(6)$ & $40(15)$ & 2.0 \\
\hline \hline
\end{tabular}




\section{Origin of the defects}

$\mathrm{Zn}$ vacancies created by irradiation with electrons are recovered at temperatures lower than $600 \mathrm{~K} .{ }^{17}$ So, the fact that the neutral Zn-vacancy-related defects $\left(\tau_{V}=230 \mathrm{ps}\right)$ in VTgrown bulk $\mathrm{ZnO}$ samples survive the $900{ }^{\circ} \mathrm{C}$ annealing in vacuum and oxygen atmospheres is an indication that the $\mathrm{Zn}$ vacancies are not isolated, but belong to a $V_{\mathrm{Zn}}-X$ defect complex.

The lowest formation energy for the $\mathrm{Zn}$ vacancy is for its double negative charge state in $n$-type material. ${ }^{22}$ However, the positrons are sensitive to the total charge of the complex, which in this case is neutral. Hence, the $\mathrm{Zn}$ vacancies observed in VT-grown are complexed with donor-type defects with no open volume, which can be either impurities or intrinsic defects. Similar neutral in-grown Zn-vacancy-related complexes have also been observed in CVT-grown ZnO. ${ }^{19}$ On the other hand, these centers behave in a different way when annealing in $\mathrm{Zn}$ atmosphere. Indeed, its concentration decreases below the detection limit of the positron annihilation spectroscopy. So, it indicates that the recovery is not caused by the dissociation and migration of $\mathrm{Zn}$ vacancies and it is due to the $\mathrm{Zn}$ vapor atmosphere at which it has been annealed. Moreover, $\mathrm{Zn}$ interstitials are very mobile at the annealing temperature. ${ }^{25}$ Therefore, $\mathrm{Zn}$ diffusion into the center must be the cause of the recovery.

The temperature behavior of the longest lifetime component indicates that the vacancy clusters are in the negative charge state. Based on the magnitude of the longest lifetime component $\left(\tau_{c l}=340 \pm 40 \mathrm{ps}\right)$, we can estimate the size of the vacancy clusters. This lifetime can be compared to those reported in $\mathrm{SiC},{ }^{26}$ where the $\mathrm{Si}$ vacancy to $\mathrm{SiC}$ bulk lifetime ratio is close to the $\mathrm{Zn}$ vacancy to $\mathrm{ZnO}$ bulk lifetime ratio. We estimate that the clusters consist of at most five missing $\mathrm{Zn}-\mathrm{O}$ pairs, as there is no reason to assume that these clusters would be $\mathrm{Zn}$ rich or $\mathrm{O}$ rich. The concentration of the vacancy clusters increases slightly in all the annealings, suggesting that new clusters are formed during the annealing by the thermal creation and migration of vacancies. The mean size of the clusters does not increase appreciably. It must be pointed out that the vacancy cluster concentration is not increasing at the expense of the Zn-vacancy-related complexes, because they are unaffected by the annealing in vacuum or oxygen atmosphere.

The ionic traps are the most abundant defects observed in our positron experiments. Their presence in as-grown samples has been typically interpreted as them being related to impurities, as the intrinsic defects tend to be mobile at rather low temperatures. However their concentration increases with the annealing in $\mathrm{Zn}$ vapor. Hence, we suggest that these negative centers (with no open volume) are related to intrinsic defects in the $\mathrm{Zn}$ sublattice.

\section{CONCLUSIONS}

We have applied positron annihilation spectroscopy to study the behavior of point defects in the annealing under different conditions of bulk $\mathrm{ZnO}$ crystals grown by VT. The positron annihilation lifetime spectra present more than one type of vacancy-type defect and ionic traps. The existence of two types of vacancy-type defect trapping positrons has been unambiguously stated subtracting one lifetime component as a source component before the lifetime analysis. The subtracted intensity has been determined using the one defect trapping model.

The vacancy-type defects are identified as neutral defect complexes involving the $\mathrm{Zn}$ vacancy and negatively charged vacancy clusters consisting of at most five missing $\mathrm{Zn}-\mathrm{O}$ pairs. The concentrations of the $\mathrm{Zn}$ vacancies are in the $0.2-0.3$ ppm range, while the concentrations of the vacancy clusters are estimated 1-2 orders of magnitude lower. The ionic traps are the most abundant defects observed in our experiments, with concentrations in the $0.5-2 \mathrm{ppm}$ range. The concentration of the Zn-vacancy-related complexes is significantly reduced after annealing in $\mathrm{Zn}$ atmosphere. The concentration of the vacancy clusters increases slightly in the annealings, possibly due to the formation and subsequent migration of vacancies on both sublattices. The concentration of the ionic traps increases after annealing in $\mathrm{Zn}$ atmosphere, which suggests that they are related to intrinsic defects in the Zn sublattice.

\section{ACKNOWLEDGMENTS}

We are thankful for the fruitful discussions with the late K. Saarinen. The work has been undertaken under Projects Nos. MAT2003-406841, MAT2004-06841, MEC872/2006, GV ACOMP06/037, and UPV/EHU-GIU06/59 and with the financial support of the Basque Government under the Researchers Formation Program. *asier.zubiaga@ehu.es

†Present address: LCMTR, Institut des Sciences Chimiques Seine Amont, CNRS, 2/8 rue Henri Dunant, 94320 Thiais, France.

${ }^{1}$ U. Özgur, Y. Alivov, C. Liu, A. Teke, M. Reshchikov, S. Dogan, V. Avrutin, S. J. Cho, and H. Morko, J. Appl. Phys. 98, 041301 (2005).

${ }^{2}$ D. C. Look, B. Caflin, Y. I. Alivov, and S. J. Park, Phys. Status Solidi A 201, 2203 (2004).

${ }^{3}$ D. C. Look, Mater. Sci. Eng., B 80, 383 (2001).

${ }^{4}$ K. Minegishi, Y. Koiwai, Y. Kikuchi, K. Yano, M. Kasuga, and A.
Shimizu, Jpn. J. Appl. Phys., Part 2 36, L1453 (1997).

${ }^{5}$ K.-K. Kim, H.-S. Kim, D.-K. Hwang, J.-H. Lim, and S.-J. Park, Appl. Phys. Lett. 83, 63 (2003).

${ }^{6}$ Y. R. Ryu, S. Zhu, D. C. Look, J. M. Wrobel, H. M. Jeong, and H. W. White, J. Cryst. Growth 216, 330 (2000).

${ }^{7}$ K. Saarinen, P. Hautojärvi, and C. Corbel, Semiconductors and Semimetals (Academic, New York, 1998), p. 209.

${ }^{8}$ R. Tena-Zaera, C. Martinez-Tomas, C. J. Gomez-Garcia, and V. Munoz-Sanjose, Cryst. Res. Technol. 41, 742 (2006).

${ }^{9}$ F. Tuomisto, V. Ranki, K. Saarinen, and D. C. Look, Phys. Rev. 
Lett. 91, 205502 (2003).

${ }^{10}$ P. Hautojärvi, Positrons in Solids, Topics in Current Physics Vol. 12 (Springer, Heidelberg, 1979).

${ }^{11}$ M. J. Puska and R. M. Nieminen, Rev. Mod. Phys. 66, 841 (1994).

${ }^{12}$ F. Plazaola, K. Saarinen, L. Dobrzynski, H. Reniewicz, F. Firszt, J. Szatkowski, H. Meczynska, S. Legowski, and S. Chabik, J. Appl. Phys. 88, 1325 (2000).

${ }^{13}$ M. J. Puska, C. Corbel, and R. M. Nieminen, Phys. Rev. B 41, 9980 (1990).

${ }^{14} \mathrm{~K}$. Saarinen, III-V Nitride Semiconductors: Electrical, Structura and Defect Properties (Elsevier, Amsterdam, 2000), p. 109.

${ }^{15}$ S. Brunner, W. Puff, A. G. Balogh, and P. Mascher, Mater. Sci. Forum 363-365, 141 (2001).

${ }^{16}$ A. Uedono, T. Koida, A. Tsukazaki, M. Kawasaki, Z. Q. Chen, S. Chichibu, and H. Koinuma, J. Appl. Phys. 93, 2481 (2003).

${ }^{17}$ F. Tuomisto, K. Saarinen, D. C. Look, and G. C. Farlow, Phys. Rev. B 72, 085206 (2005).

${ }^{18}$ G. Brauer, W. Anwand, W. Skorupa, J. Kuriplach, O. Melikhova, C. Moisson, H. von Wenckstern, H. Schmidt, M. Lorenz, and M. Grundmann, Phys. Rev. B 74, 045208 (2006).

${ }^{19}$ F. Tuomisto, A. Mycielski, and K. Grasza, Superlattices Microstruct. (to be published).
${ }^{20}$ S. Dannefaer, Phys. Status Solidi A 102, 481 (1987); J. Mäkinen, C. Corbel, P. Hautojärvi, P. Moser, and F. Pierre, Phys. Rev. B 39, 10162 (1989); K. Saarinen, J. Nissilä, H. Kauppinen, M. Hakala, M. J. Puska, P. Hautojärvi, and C. Corbel, Phys. Rev. Lett. 82, 1883 (1999).

${ }^{21}$ S. Kuisma, K. Saarinen, P. Hautojärvi, C. Corbel, and C. LeBerre, Phys. Rev. B 53, 9814 (1996); J. Gebauer, M. Lausmann, F. Redmann, R. Krause-Rehberg, H. S. Leipner, E. R. Weber, and P. Ebert, ibid. 67, 235207 (2003).

${ }^{22}$ A. F. Kohan, G. Ceder, D. Morgan, and Chris G. Van de Walle, Phys. Rev. B 61, 15019 (2000).

${ }^{23}$ R. M. Nieminen and J. Laakkonen, Appl. Phys. 20, 181 (1979).

${ }^{24}$ K. Saarinen, S. Hautakangas, and F. Tuomisto Phys. Scr. T126, 105 (2006).

${ }^{25}$ Y. V. Gorelkinskii and G. D. Watkins, Phys. Rev. B 69, 115212 (2004).

${ }^{26}$ G. Brauer, W. Anwand, P. G. Coleman, A. P. Knights, F. Plazaola, Y. Pacaud, W. Skorupa, J. Störmer, and P. Willutzki, Phys. Rev. B 54, 3084 (1996); G. Brauer, W. Anwand, P. G. Coleman, J. Störmer, F. Plazaola, J. M. Campillo, Y. Pacaud, and W. Skorupa, J. Phys.: Condens. Matter 10, 1147 (1998); R. Aavikko, K. Saarinen, F. Tuomisto, B. Magnusson, N. T. Son, and E. Janz'en Phys. Rev. B 75, 085208 (2007). 